\title{
Photoelectric Polarimetry and the Gas Pixel Detector Yesterday, Today and Tomorrow
}

\author{
Enrico Costa ${ }^{1,2}$ (D) \\ 1 National Institute for Astrophysics (INAF)-Institute for Space Astrophysics and Planetology (IAPS), \\ via del Fosso del Cavaliere 100, 00133 Rome, Italy; enrico.costa@iaps.inaf.it or enrico.costa@asi.it \\ 2 Agenzia Spaziale Italiana, Via del Politecnico, s.n.c., 00133 Rome, Italy
}

Received: 28 May 2018; Accepted: 10 July 2018; Published: 16 July 2018

\begin{abstract}
Since the very beginning of X-ray Astronomy, polarimetry has been suggested as a tool of diagnostics, of great potentiality. While almost all measurements of $X$-rays were based on detectors using the photoelectric effect, the first attempt to perform polarimetry were based on Compton scattering and Bragg diffraction. The use of photoelectric effect also for polarimetry has been hypothesized and attempted for many years but never accomplished. Only 40 years from the start of X-ray astronomy, the Gas Pixel Detector (GPD) was developed, compatible with an X-ray optics, and capable of measuring energy, time, position and polarization simultaneously. Only after 20 more years, the Imaging X-ray Polarimetry Explorer, based on the GPD detectors, will be launched. I present the story of the development of photoelectric polarimetry that arrived to the Gas Pixel Detector, and discuss the possible future evolutions.
\end{abstract}

Keywords: X-ray polarimetry; instrumentation; polarization

\section{The Quest for X-ray Polarimetry}

Scientists devoting a significant amount of their time and with a continuity not far from obstinacy to X-ray Polarimetry have been a well confined subset of the X-ray Astronomy community.

An original promoter of this small community was Vitaly Ginzburg. Since the discovery of a strong $\mathrm{X}$-ray emission from the Crab, Ginzburg foresaw the relevance of a measurement of polarization to discriminate whether the synchrotron was still the dominant emission process and the implication on the acceleration processes.

Both Robert Novick and Eliuh Bolt reported to me that their very early efforts to measure polarization from X-ray sources were performed under the pressure of Ginzburg. It is noticeable that Ginzburg did not do personally any specific work on these predictions. Soon after the high accuracy measurement of polarization from the Crab with OSO-8 by the Columbia Team [1], Ginzburg declared his belief on the future role of X-ray polarimetry [2]. However, many scholars have worked on this subject. This did not occur with continuity because it is hard to keep a high attention to a scientific topic not supported by data for two scores of years.

However, it is well established that many emission processes and physical interaction involve a high polarization of photons also in X-rays.

The early stages of this discipline have been reviewed by Robert Novick, who got the leadership since the very beginning $[3,4]$. He attempted the Thomson scattering around $90^{\circ}$ without substantial result, also because of the choice of ScoX1 as a target and the difficulty to control systematics and the range tuned on higher energies, where less photons are available [5]. In practice, the only viable technique to perform X-ray polarimetry was the Bragg diffraction around $45^{\circ}$. This technique, originally proposed by Herbert W. Schnopper [6] was fully exploited by the group of Columbia University [7] headed by Robert Novick. Leicester University was operating in the same field with an instrument aboard 
ARIEL-5 satellite [8], also based on Bragg diffraction. The success of Bragg, based on very mature techniques, somehow discouraged the efforts to develop alternative approaches.

In X-ray Astronomy, as in other fields of Space Science, there have always been plenty of proposed missions. Polarimetry instruments aboard missions with complex payloads or missions dedicated to polarimetry have been proposed in various epochs. The presence of theoretical papers in the literature has been correlated to the expectation of having one of these missions approved.

Another peculiarity of X-ray polarimetry is that there have been two experiments approved and never flown for different reasons. The Stellar X-ray Polarimeter was approved, built and calibrated, but the supposed mission, Spectrum X Gamma, was never flown due to the collapse of Soviet science. The Gravity and Extreme Magnetism Small Explorer (GEMS) was approved by NASA in 2009 and cut in 2012, due to schedule and budget problems. In addition to all the general difficulties to have an approved experiment, these two missions discouraged for many years the submission and the approval of new proposals.

In the present paper, I discuss the development of photoelectric polarimetry in the classical 2-8 keV range, in the frame of the evolution of competing techniques. When, at the end, I introduce the potential extension of the technique to the Hard X-ray range, I omit any discussion or comparison with other techniques or experiments in this same range, because this would require a significant increase of the dimensions of the paper.

\section{The Prehistory of GPD: The Cloud Chamber}

By combining the available data and current theories scholars as Auger, Kirkpatrick, Kirchner and Richardson arrived, in the years spanning from 1925 to 1930, to state that the angular distribution of photoelectrons is of the type:

$$
P(\varphi, \vartheta) \propto \cos ^{2}(\varphi) \sin ^{2}(\vartheta)
$$

where $\vartheta$ is the angle with respect to the photon direction and $\varphi$ is the azimuth angle with respect to the electric field [9]. There is further bending forward increasing with energy, accounting to preserve the momentum, but, given the energies involved, this is not relevant for this discussion.

Once this point is assessed, the angular distribution of photoelectrons around the direction of the incoming beam, can be used to perform X-ray polarimetry. The information on linear polarization is encoded in the direction of the photoelectron ejected as consequence of the interaction. From a microscopic point of view, the phenomenology is quite complex. The electron is scattered, slowed by ionizing and non ionizing collisions, and eventually stopped. Ionizations track the path of the photoelectron from the creation to the end. Given the energies involved, the energy losses are in the low energy side of the Bethe Bloch curve, where the liner energy loss is inversely proportional to the kinetic energy. In addition, the scattering probability is inversely proportional to the square of the kinetic energy. The track is more straight and less dense at the beginning, then is scattered with more frequency and is continuously increasing the linear energy loss and thence the ionization density. Most of the energy is released at the end of the path, where the direction is totally randomized, while the information on polarization is all contained in the other side of the track near the absorption point. If we want to exploit this information, we must visualize as far as possible the ionization track, identify the start and the end of the track and reconstruct the direction close to the absorption point.

Actually, this does not requires modern technology, but has been performed since almost one century and has its place in History of Physics. In the 1920s, Pierre Auger studied the physics of the photoelectrons ejected by X-rays in gases by means of a cloud chamber filled with gases at different pressures and compositions [10]. From his images, we can see in Figure 1 a few examples of tracks. The tracks within low atomic number gases are more straight. A cloud chamber with stereoscopic read out was in fact used to study the angular distribution of photoelectrons by Auger. On the contrary Bothe extracted photoelectrons from thin targets, and measured the angular distribution by rotating a small Geiger counter around the target [11]. These can be considered the two alternative methods for photoelectric polarimetry also in the future: a single medium acting both as converter and detector and 
a detector separated from the converter. In the first method, the information on the first interaction is also preserved. In the second method, for which the converter is typically a passive material, some information is lost. Both Auger and Bothe knew in advance the energy of the X-ray photons they injected in the apparatus. This is not, unfortunately, the case for astrophysical observations. In the second approach the energy lost in the converter is unknown. This introduces an ambiguity in the interpretation of the results and makes the approach less attractive. However, the first method faces the problem that the track details extension is orders of magnitude smaller than X-ray absorption length. The read-out of an effective polarimeter must include thousands or, better, millions of pixels. At the time of Auger this was only feasible with photographic methods.

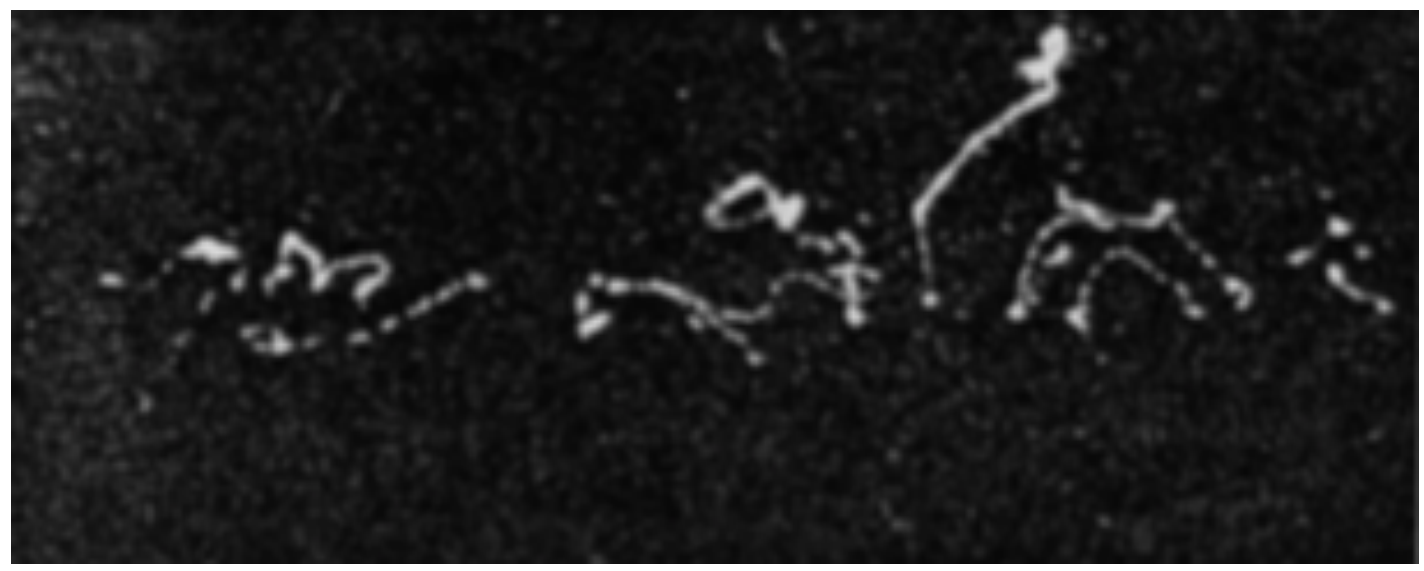

Figure 1. A cloud chamber image from the paper by M. Pierre Auger in 1925. The picture shows photoelectron tracks from photons of $30 \mathrm{keV}$ absorbed in a gas mixture based on Argon [10].

What we needed to perform an efficient photoelectric polarimetry is a modern cloud chamber. The Gas Pixel Detector (GPD) is a modern implementation of this concept, with performances sometimes better and sometimes worse than the archetypical instrument. Starting from the negative side:

- The GPD is not truly three-dimensional, as a cloud chamber with stereoscopic read-out. However, the information on linear polarization is carried by the azimuth angular distribution on the plane perpendicular to the optical axis. Thence, in the case of an ideal read-out, the information on polarization is totally preserved by the projection of the track on this plane. In practice, this is not true because of the blurring and imperfect reconstruction, which is higher for electrons ejected forward or backward, but it comes out to be acceptable in practice.

- The photoelectric technique is specially powerful in astrophysics when the detector is positioned in the focus of an X-ray optics system. In this case, the space resolution cannot be improved above a certain limit because of the effect of inclined penetration in the gas of finite thickness. The interaction points have a certain distribution above and below the focal plane with an unavoidable blurring. The penetration angles are not a free parameter of the design, since they are determined by the band-pass of the optics which is more or less defined by the energy band.

Conversely, 90 years of technological developments result in a few but substantial improvements

- $\quad$ Electronic read-out.

- Prompt read-out.

- Self triggering.

- Mass, dimensions and consumption compatible with the focal plane of a, space based, X-ray telescope. 


\section{From the Concept of Photoelectric Polarimetry to the GPD}

The path from the cloud chamber to the GPD was neither fast nor simple. It is worth mentioning some attempts. First, although the interest on imaging gas detectors with electronic read-out was clear also for several ground based experiment, a real investment toward an instrument useful or astrophysical purposes could not be even conceived, of course, before the birth of X-ray Astronomy. Arriving to derive an information from the track of the photoelectron means to have the capability of extracting details which are a fraction of the track extension. This must be combined with a reasonable efficiency of detection.

To follow the development of photoelectric polarimetry, we must mention that X-ray Astronomy has a well defined turning point, namely the passage from collimated instruments to detectors in the focal plane of grazing incidence telescopes. The first attempts of polarimetry clearly belong to the era of spinning satellites and collimated detectors. I am aware that, in more than one institution, attempts were made to detect coincident signals in contiguous wires of proportional counters. The idea was to detect coincident signals between contiguous anodes from photons entering the detector from a direction parallel to the anodes. The rate of these coincidences would be modulated with the phase of the spinning of the satellite. As far as I know, no conclusive result was achieved and some of these efforts are not documented by publications. The only example I find in the literature [12] of this kind of testing is dated 1970. Another detector for X-ray Astronomy exploiting the photoelectric effect is described in a paper of the same year by Sanford et al. [13]. This team of researchers of Mullard Space Science Laboratory (MSSL) tried a method based on pulse shape discrimination in proportional counters. Since the beginning of X-ray Astronomy it was found that X-rays produce pulses faster than background events of the same amplitude. A window in the pulse rise-time was effective to discriminate a large fraction of the background. This was interpreted as because a photoelectron, which is at the end of range, produces a ionization track confined in a very small region. The electrons drift all together and arrive to the anode at the same time so contributing to a fast pulse. On the contrary, pulses of background are produced by electrons not at end of range with a minor linear ionization. In particular minimum ionizing particles are depositing the same amount of charge in longer segments. Electrons are produced at different distances from the anode and arrive to the anode at different times. Eventually the total pulse is the superimposition of pulses starting at different times. Consequently, the background pulses are slower and this make possible an electronic discrimination. Photons with polarization parallel to the anode produce a track whose electrons have a minor spread in distances with respect to electrons produced by a track polarized perpendicularly to the anode. Thus, photons with polarization parallel to the anode would originate signals in average faster than those with polarization perpendicular. In fact, the MSSL team found a difference. However, this interesting result was not followed by any further activity, also because the found difference was very little. A team from Osaka University tried many years later to perform similar measurements by using Xe filled proportional counters. Results [14] were not adequate to suggest that this could be the way to a practical implementation.

These preliminary attempts were based on the search of a simple phenomenology, typically a one-parameter datum, and searching for its modulation with the spinning phase. Meanwhile, the bragg diffraction provided a method to measure polarization with a very limited sensitivity but with an excellent robustness so these attempts to use the photoelectric effect for polarimetry were abandoned. Many years later, a strong step forward was done again with the studies for search for a real imaging detector based on photoelectric effect. A team of the Marshal Space Flight Center (MSFC) headed by Brian D. Ramsey was the first to achieve a first important result. The idea was to use a proportional counter filled with a mixture containing Argon and a well known photoemitting gas, the trimethylamine (TAE). The electrons of the track drift to a multiplication grid where, under the effect of the electric field, emit optical photons. In practice, the track becomes luminous for a while and can be imaged with an optics and a Charge Coupled Device (CCD). The results are impressive [15] and are in the right direction, namely a high resolution mapping of the track. However, it was still 
far from the target of a practical application in a real experiment. The method was demonstrated for hard X-rays but could be only applied in practice in the range between the energy where the atmosphere becomes transparent at balloon altitude and the energy where the gas mixture, based on Argon, and with a limited absorption gap becomes transparent itself. Too few photons for polarimetry in a range of energy with a huge background. The next step would be definitely the extension of the technique to lower energies, moreover that at that time the multi-layer optics for hard X-rays were still in a conceptual stage.

\section{The Present of the GPD}

\subsection{The One-Dimension Prototype}

The device developed at MSFC was exploiting the high resolution of the imaging optical devices. However, at the same time, the imaging capabilities of the gas detectors was also improving, but mainly in the 1-D versions. This was the basis of a convergence of a team of X-ray Astronomy at IAS-CNR in Rome headed by myself and the team of gas detectors at Pisa INFN headed by Ronaldo Bellazzini. The idea was to use the micro-gap detectors, an evolution of the micro-strip detectors, recently developed by the Pisa Team, with $200 \mu \mathrm{m}$ pitch, filled with a low atomic number gas to search for a, polarization dependent, asymmetry in the track length.

To minimize the scattering and have straighter tracks, the team used a mixture based on Neon. The source of polarized photons was obtained by scattering 5.4 and $8.0 \mathrm{keV}$ photons from two generators on a lithium target with input and output collimator constraining the scattering angle around $90^{\circ}$. As unpolarized source, the $5.9 \mathrm{keV}$ photons from $F e_{55}$ were used. In Figure 2, the data show a clear difference in the length of tracks created with photons polarized perpendicularly to the gaps and those created by photons with parallel polarization. No such difference existed with unpolarized photons. This result was published on 1995 [16]. The tracks were one-dimensional and had not the impressive quality of the MSFC detector, but the micro-gap detector had a very simple, self triggered, electronic readout without the slowness of the CCD analysis and the encumbrance of the optics. Finally, the detector worked at $5.4 \mathrm{keV}$ and thence was entering the range of operations of classical X-ray optics. After a first quick analysis of these data in 1995 a new analysis was performed five years later [17]. Data were compared with a Monte-Carlo code for micro-analysis. In addition, the possible applications to an experiment of X-ray Astronomy were discussed. The sensitivity to polarization is usually expressed with an instrumental parameter the modulation factor as defined in [4]. A factor of $7 \%$ and $11 \%$ was found at 5.4 and $8.0 \mathrm{keV}$, respectively. The latter could be possibly increased to $20.5 \%$ by cuts applied on the basis of the cluster size. The later bi-dimensional implementations would be significantly better but, when compared with the previous attempts with gas counters and charge readout, this was already a substantial achievement.

This work had achieved several points:

- The one-dimensional projection of the photoelectron track could be visualized.

- A neon and organic gas based mixture was viable. The phenomenology could be well described with a good Monte Carlo code.

- $\quad$ The found modulations were not yet suited to do astrophysical polarimetry but not far from a good goal.

It was not bad as a first attempt. The paper conclusions are a true manifest for a photoelectric polarimetry and states the need of full pixel detector with an algorithm to detect the interaction point and the photoelectron direction 


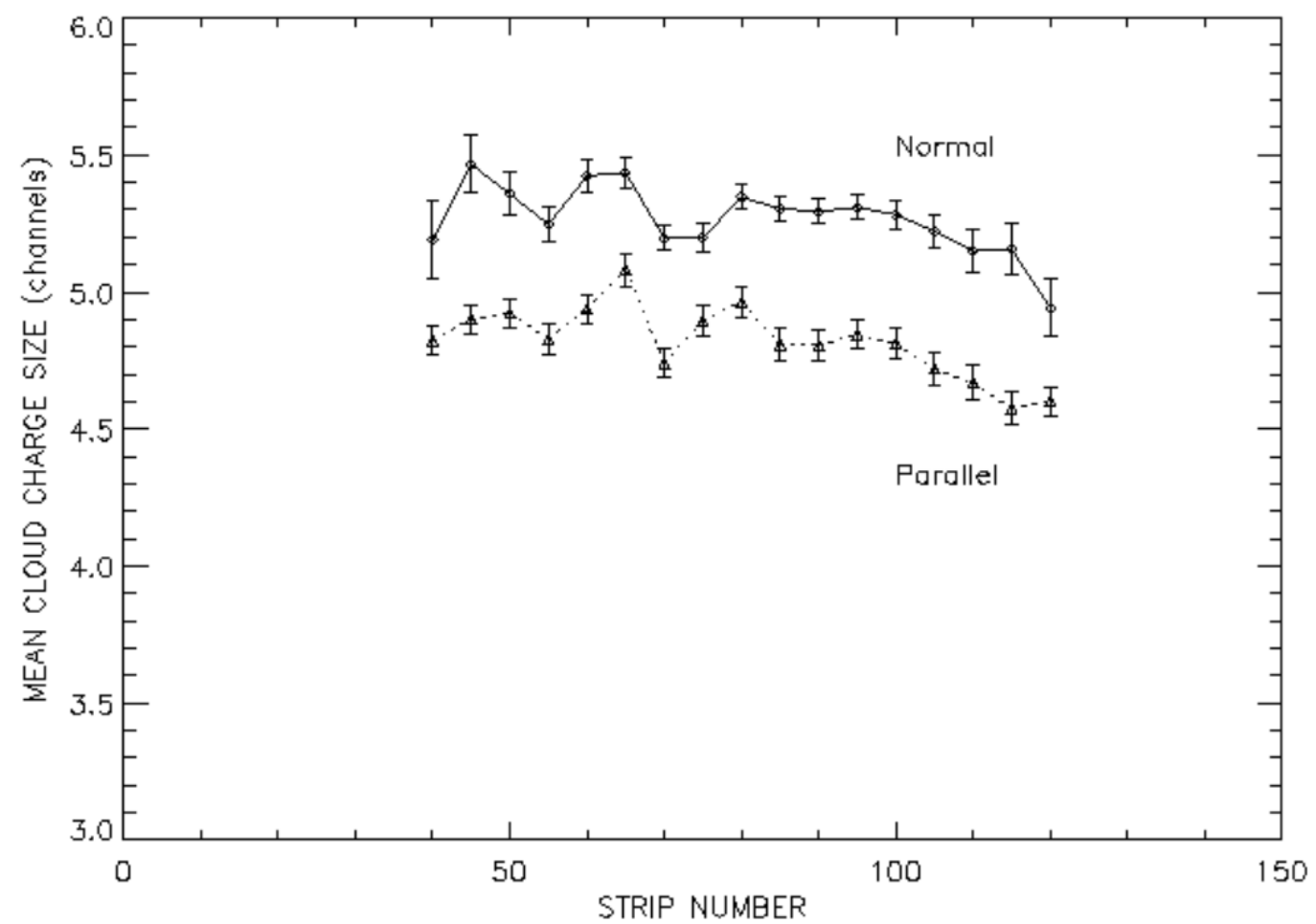

Figure 2. The first detection of the effect of polarization by the Pisa and Roma teams with a micro-gap detector filled with a Ne-Dimethyl Ether (DME) mixture. The tracks produced by photoelectrons created by photons polarized perpendicular to the strips are definitely longer than those created by photons polarized parallel to the strips. The pitch of the strips is $200 \mu \mathrm{m}$. The figure shows that the effect is the same through the whole detector.

Such a device could be put in the focus of an X-ray telescope and would allow for simultaneous measurement of position, energy spectrum and energy-resolved linear polarization for all the sources in the field of view.

The publishing process was very late and slow and the paper was printed only a few months before the publication of the paper on the GPD. However, we can state that all the basic concepts and tools of physics, statistics, simulation tool and astrophysics for the GPD development are already there.

However, passing from the one-dimensional strip geometry to the two-dimensional pixel geometry needed a major step in technology. This is what the Pisa group achieved in 2001 arriving to the first prototype, which started a new era for astronomical X-ray Polarimetry.

The development of GPD is well documented in the literature by many publications giving details on the various versions of the device. I want here to stress the point that, although the development was very fast, it is clearly articulated into three steps, which are described below. All three steps share some basic technical solutions:

- A gas cell with an insulating body and a conductive frontal window.

- An electric field parallel to the optical axis to drift the electrons of the track to a multiplication stage.

- A Gas Electron Multiplier (GEM) to perform a proportional multiplication of the electrons to amplify the track without destroying the spatial and energetic information.

- An electric field to drift the multiplied electrons from the bottom of the GEM to a sense plane.

- A plane of metal pads distributed on a honeycomb pattern to act as anodes to collect the charge multiplied in the gas above the pad itself.

- A dedicated front end electronic chain independent for each pad. 


\subsection{The Pixel Break-Through}

A first prototype based on trigger by GEM and collection plane built with multi-layer printed circuit technology. The pad pitch was $150 \mu \mathrm{m}$. The GEM pitch was $60 \mu \mathrm{m}$. Signals were routed horizontally to a set of application specific integrated circuit (ASIC) chips, relatively far from the gas cell.

However, it should be clear that the technology applied had serious limitations that would make a flight device still cumbersome and with severe limitations.

- The technique of multi-layer printed circuit board (PCB) would never allow for a number of pixels larger than $\simeq 1000$ and anode pads below $100 \mu \mathrm{m}$. Given the length of the tracks, this would limit the field of view of any application of the GPD and, in practice, to point-like sources. However, the diffusion in the drift was compatible with a finer readout.

- The location of the front end electronics far from the anode pads implies a relatively high electronic noise and a cross section of the instrument much larger than the gas cell itself.

Notwithstanding these limitations, data from this prototype showed that imaging photoelectric polarimetry was, at last, feasible. The modulations found were adequate to provide a dramatic step forward in sensitivity and in the control of systematics [18]. As the citation record shows, the paper also represents a vigorous re-start of the theoretical analysis and prediction for this subtopics of High Energy Astrophysics.

\subsection{From $P C B$ to ASIC}

The next step performed by the Pisa team was the development of a very large scale integration (VLSI) ASIC chip. The first layer would host the multi-pad plane acting as both bottom of the detector and anode array. In the inferior layers, just below the projection of each pad an analog and digital electronic chain handles the signal from each pad. Under trigger from the GEM, the analog signal is held. The content of all pixels is Analog to Digital (A/D) converted. The digitized image is analyzed to detect the cluster of pixels with non-null content, associated to the track, and identify the starting point and determine the starting direction of the photoelectron.

The revolution of this step is that everything collapses inside the same cell containing the gas. The dimensions become incredibly small. The electronics contiguous to the pad has a very low noise. The whole system benefits from the stability and the radiation hardness of the high integration VLSI.

The chips of this stage are two, one with 2000 pixels [19] and the other with 22,000 pixels [20]. The pitch of the pad was reduced to $80 \mu \mathrm{m}$. These two devices are still based on a trigger from the Gas Electron Multiplier starting the download and the conversion of the content of all the pixels of the chip.

\subsection{The Self-Triggering}

The last passage was a further increase of the area with a chip of 105,000 pixels, with a pitch of $50 \mu \mathrm{m}$, for a total of about $2 \mathrm{~cm}^{2}$ [21]. Given that, to minimize noise, the A/D conversion frequency cannot exceed $10 \mathrm{MHz}$, the download of the whole image and the post facto search for the track would result into an unacceptable dead time. Thence, the chip was designed with a capability of self-triggering. A Region of Interest is defined around the pixels wich have triggered. Only the content of the pixels inside the region of Interest are downloaded and converted. Therefore, for X-rays in the range, the number of pixels downloaded is typically less than $1 \%$ of the total. Eventually, this new chip was mounted inside a sealed detector with a thin $(50 \mu \mathrm{m})$ beryllium window [22]. The very new technology was, therefore, combined with a very traditional set up of detector manufacture for long duration operations. These first sealed prototypes had the total mass of $50 \mathrm{~g}$. The three generations of the chip are shown in Figure 3. 


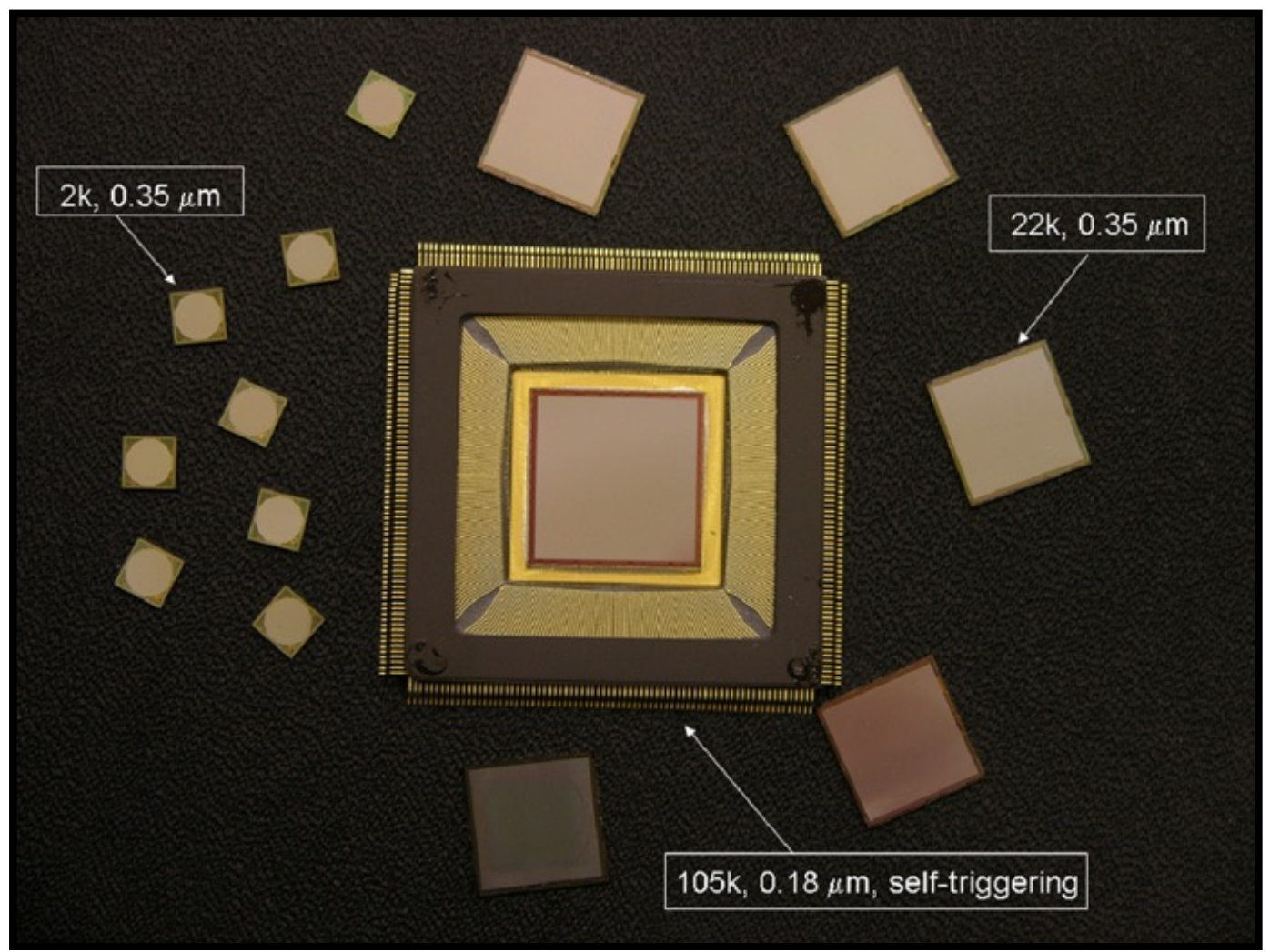

Figure 3. The comparison of the three generations of ASIC chips, developed by the Pisa group, starting from 2000 to arrive to more than 100,000 pixels. The chip of $105 \mathrm{k}$ pixels is shown bonded to its keramic package [22].

Meanwhile, the Roma team was improving the testing and measurement capability, by building a set of monochromatic X-ray sources polarized to near $100 \%$ by bragg diffraction and very compact capillary plates as collimators [23]. The sources combined with collimators, diaphragms and micrometric movements allows for a detailed characterization of the detectors.

With these developments a detector is at last available to allow for polarimetry the revolution actuated by Einstein Mission on imaging and spectroscopy. This chip has been the basis of all the proposals of mission, included the Imaging X-ray Polarimetry Explorer. This mission was approved on 2017 by NASA to be launched in the beginning of 2021. It includes three telescopes with GPDs in the focus. It will be definitely the first satellite mission totally dedicated to low energy X-ray polarimetry.

\subsection{The Alternatives: Time Projection}

It is worth mentioning the fact that a team of Yamagata University headed by H. Sakurai was developing in the late 1990s a gas detector in some sense similar to that of Brian Ramsey, with a mixture including Argon and photoluminescent gas trimethylamine (TMA), performing the multiplication by an electric field between two faces of a capillary plate and having a CCD as optical read-out. In 1999, they published images of photoelectrons at 15 and $22 \mathrm{keV}$ and mention the possible implications for polarimetry [24]. In 2004, they published the first polarimetric application of the detector [25]. They also tested the use of $\mathrm{CF}_{4}$ gas. The whole is more cumbersome of the GPD but is, likely, the best published result beside GPD in the same time horizon.

A few years after the announcement of the GPD, a team from Goddard Space Flight Center, headed by Keith Jahoda, developed a new detector for photoelectric X-ray polarimetry [26]. Similar to the GPD, the TPC is based on visualization of the track originated by the photoelectron in a low atomic 
number $(Z)$ gas. The electrons in the track are drifted from an electric field perpendicular to the optical axis. After amplification from a GEM, they are collected from an anode made of strips parallel to the optical axis. The strip determines one of the coordinates of the image. The other coordinate, along the drift direction, is determined with the time-projection technique.

The main strength is that the drift direction is different from the absorption direction and no trade-off between efficiency and diffusion is needed. The TPC could be in principle very efficient.

A major weakness of this technique is that by drifting the electrons of the track in a direction perpendicular to the optical axis the symmetry around this axis is lost. Azimuth dependent systematics are introduced and can only be compensated through the rotation of the instrument (or of the satellite).

Another major weakness is that the image of the projection of the track is well reconstructed but the position is undetermined in the drift direction. This weakness is common to all the imaging detectors based on drift, whenever the origin of time is not provided externally (e.g., the bunch time of the accelerator, or an external coincidence for particles). This is the reason they are usually not employed for photons. In the case of GEMS, the effect, combined with rotation results in the fact that polarimetry is performed of the photons falling within a relatively large field of view, with a diameter of around 10 arc-minutes. This means that the background detected in that large region is also to be accounted for, while, for the GPD, for point-like sources, it is only that within the point spread function.

This detector was foreseen for the GEMS mission, first approved, and later suppressed, by NASA.

\section{The Future of GPD}

The GPD is definitely the instrument with the right timing to match the maturity of Polarimetry as a sub-topics of X-ray Astronomy. The Imaging X-ray Polarimetry Explorer [27] is based on the GPD and will disclose this almost new window for most of classes of X-ray sources. IXPE is a small mission, very fast in the implementation and relatively cheap. The missions that will follow can expand this discovery space under different aspects. In some cases, this will be based on an evolution of the GPD itself:

- Larger collecting area of the optics to detect weaker sources and to extend the sample on each class.

- Longer focal length of the optics to improve angular resolution, given that the space resolution is not easy to improve due to the blurring associated with inclined penetration and finite thickness.

- Contemporary presence of other instruments for simultaneous spectroscopy and timing, providing an additional tool to do deep physics on sources which show a high variability in luminosity, spectrum and, very likely, polarization.

- Change or extension of the energy band.

A GPD was for many years foreseen aboard XEUS [28] and IXO. This would satisfy the first two points, i.e., the limit sensitivity and the angular resolution. On the other side, these large observatories were conceived with a single telescope and different instruments to be alternatively positioned in the focus, the polarimetry could expect only a modest share (of the order of $5 \%$ ) of the total observing time. Therefore, the goal of significantly extending the sample would not be fulfilled by such an observatory. In any case, the team of ATHENA, the last version of this concept, eventually selected by ESA, decided not to include a polarimeter in the baseline payload. In the present landscape, given that the X-ray Imaging and Polarimetry Experiment is in this moment out of the competition for M4 ESA mission, we can expect that the Chinese mission Enhanced X-ray Timing and Polarimetry Mission (eXTP) will host a set of telescopes with GPDs in the focus and with a total area much larger than IXPE, hopefully of almost one order of magnitude. Polarimeters aboard eXTP would also benefit from the contemporary presence of instrumentation capable of performing spectra and timing with high sensitivity. As far as the GPD is concerned, only moderate improvements are needed, mostly to allow for a reduction of dead time as well as higher counting rates.

The situation is different for the extension of the band. An extension to the lower energies is possible by substituting the Be window with a plastic or $A l N_{3}$ one and possibly by reducing the 
pressure of the filling gas. In any case, the gain at low energies would be moderate while the loss of efficiency at higher energies would be substantial.

The analysis is much more encouraging on the side of the higher energies. Simulations show that GPD can work effectively as polarimeters with Argon based mixtures, with a pressure up to $3 \mathrm{~atm}$ and an absorption gap of the order of $30 \mathrm{~mm}$ [29]. The team built a first prototype of such a medium energy polarimeter and had to face a problem of uniformity of electric field. To have more freedom to increase the thickness of the absorption gap they had to make wider electrodes. Thus, they changed the design. Nowadays, this larger body is also adopted for lower energies, included the detectors for IXPE.

A second prototype was built, based on this new geometry. First, testing at IAPS showed that it was working as predicted by simulation, but a leak problem turned the prototype into something not operative in a few weeks [29]. If we consider that:

- The credibility of simulations increases with energy.

- In gas detectors, there is much more experience in the Argon based mixtures than in pure Dymethyilether (DME).

- Testing of the last prototype has only failed under the aspect of leak tightness, a problem not trivial but faced and solved many times in the past, also for detectors more sensitive to gas purity as Gas Scintillation Proportional Counters.

We can assume, with reasonable confidence, that this extension of the GPD performance to higher energies, should work, and arriving to the Readiness Level needed for a mission proposal should be feasible in a reasonable time.

Now, a good question is whether it is it worthwhile to conceive a mission hosting at least one of these telescopes that the team names Medium Energy Polarimeter (MEP).

In fact, the change of the band is more a shift than an extension. The Auger electron in Argon, which has an isotropic distribution, has an energy of about $3 \mathrm{keV}$. The photoelectron must, at least, exceed this energy to preserve a minimum of the anisotropy, that carries the polarimetric information. Let us say that $8-25 \mathrm{keV}$ is a practical range of operation. There are a few, but not trivial at all, topics where the MEP would represent a great step forward:

- The Supernova Remnants (SNR) at energies below $8 \mathrm{keV}$ are dominated by a huge unpolarized thermal emission. For sure at higher energies, the study of the non thermal emission and thence of the shock front will be much more effective.

- No cyclotron line has been so far detected below $9 \mathrm{keV}$. Only for a very fortunate combination of circumstances, one could be studied with IXPE. A good fraction of them (from one third to one half) falls below $30 \mathrm{keV}$. They are an excellent target for a MEP.

- A significant role of reflection is foreseen in many scenarios of High Energy Astrophysics. Beside the spectral signatures (Fe lines, Compton bump) and the timing signatures (reverberation), important and distinct polarization signatures have been predicted in binaries and Active Galactic Nuclei. Some measurements for a few of the brightest sources will be performed by IXPE. However, this is the domain of an instrument tuned on higher energies.

- As in the case of SNR, the spectrum of solar flares is also dominated by a huge thermal emission but above $10 \mathrm{keV}$ the non thermal emission is prevailing. Fabiani [30] demonstrated that even a single MEP, without any optics, would be more sensitive to polarization of solar flares than any of the experiments flown so far.

In he field of stellar polarimetry, I expect that a combination of Low Energy $(2-8 \mathrm{keV})$ and Medium Energy (6-25 keV) polarimeters could be hosted aboard the same mission. The addition of a focal plane scattering polarimeter (20-80 keV) could set up a combination sensitive from 2 to $80 \mathrm{keV}$, with a balanced sensitivity in the three ranges [31]. For the time being, let us stay on the short term future and wait for the IXPE results [27]. 
Funding: This review research received no external funding

Acknowledgments: The development of GPD was primarily supported by the National Institute of Nuclear Physics (INFN), National Institute for Astrophysics (INAF) and the Italian Space Agency (ASI).

Conflicts of Interest: The author declares no conflict of interest.

\section{References}

1. Weisskopf, M.C.; Cohen, G.G.; Kestenbaum, H.L.; Long, K.S.; Novick, R.; Wolff, R.S. Measurement of the X-ray polarization of the Crab Nebula. Astrophys. J. 1976, 208, L125-L128. [CrossRef]

2. Ginzburg, V. Physique Theorique et Astrophysique; Edition Mir: Moscow, Russia, 1978; pp. 404-405.

3. Novick, R. Stellar and Solar X-ray Polarimetry. In IAU Colloq. 23: Planets, Stars, and Nebulae: Studied with Photopolarimetry; Gehrels, T., Ed.; University of Arizona Press: Tucson, AZ, USA, 1974; p. 262.

4. Novick, R. Stellar and Solar X-ray Polarimetry. Space Sci. Rev. 1975, 18, 389-408. [CrossRef]

5. Novick, R.; Wolff, R.S. A Large Area Thomson-Scattering Stellar X-ray Polarimeter. In New Techniques in Space Astronomy; Labuhn, F., Lust, R., Eds.; IAU Symposium; Springer: Dordrecht, The Netherlands, 1971; Volume 41, p. 159.

6. Schnopper, H.W.; Kalata, K. Polarimeter for Celestial X rays. Astron. J. 1969, 74, 854. [CrossRef]

7. Weisskopf, M.C.; Berthelsdorf, R.; Epstein, G.; Linke, R.; Mitchell, D.; Novick, R.; Wolff, R.S. A graphite crystal polarimeter for stellar X-ray astronomy. Rev. Sci. Instrum. 1972, 43, 967-976. [CrossRef]

8. Pounds, K.A. Measurements of the Polarisation, Spectra and Accurate Locations of Cosmic X-ray Sources. In New Techniques in Space Astronomy; Labuhn, F., Lust, R., Eds.; IAU Symposium; Springer: Dordrecht, The Netherlands, 1971; Volume 41, p. 165.

9. Compton, A.H.; Allison, S.K. X-rays in Theory and Experiment, 2nd ed.; D. Van Nostrand: New York, NY, USA, 1935; pp. 564-582.

10. Auger, M.P. Sur l'effect photoelectrique composè. J. Phys. Radium 1925, 6, 205-208, . [CrossRef]

11. Bothe, W. Die Emissionsrichtung durch Röntgenstrahlen ausgelüster Photoelektronen. Zeitschrift Physik 1924, 26, 59-73. [CrossRef]

12. Riegler, G.R.; Garmire, G.P.; Moore, W.E.; Stevens, J.C. A low-energy X-ray polarimeter. Bull. Am. Phys. Soc. 1970, 15, 635. [CrossRef]

13. Sanford, P.W.; Cruise, A.M.; Culhane, J.L. Techniques for Improving the Sensitivity of Proportional Counters Used in X-ray Astronomy. In Non-Solar X-and Gamma-Ray Astronomy; Gratton, L., Ed.; IAU Symposium; Springer: Dordrecht, The Netherlands, 1970; Volume 37, p. 35.

14. Hayashida, K.; Miura, N.; Tsunemi, H.; Torii, K.; Murakami, H.; Ohno, Y.; Tamura, K. X-ray polarimetry with a conventional gas proportional counter through rise-time analysis. Nucl. Instrum. Methods Phys. Res. A 1999, 421, 241-248. [CrossRef]

15. Austin, R.A.; Ramsey, B.D. Optical imaging chamber for X-ray astronomy. Opt. Eng. 1993, 32, $1990-1994$. [CrossRef]

16. Soffitta, P.; Costa, E.; Morelli, E.; Bellazzini, R.; Brez, A.; Raffo, R. Sensitivity to X-ray polarization of a microgap gas proportional counter. In X-ray and EUV/FUV Spectroscopy and Polarimetry; Fineschi, S., Ed.; SPIE: Bellingham, WA, USA, 1995; Volume 2517, pp. 156-163,

17. Soffitta, P.; Costa, E.; di Persio, G.; Morelli, E.; Rubini, A.; Bellazzini, R.; Brez, A.; Raffo, R.; Spandre, G.; Joy, D. Astronomical X-ray polarimetry based on photoelectric effect with microgap detectors. Nucl. Instrum. Methods Phys. Res. A 2001, 469, 164-184. [CrossRef]

18. Costa, E.; Soffitta, P.; Bellazzini, R.; Brez, A.; Lumb, N.; Spandre, G. An efficient photoelectric X-ray polarimeter for the study of black holes and neutron stars. Nature 2001, 411, 662-665. [CrossRef] [PubMed]

19. Bellazzini, R.; Angelini, F.; Baldini, L.; Bitti, F.; Brez, A.; Ceccanti, M.; Latronico, L.; Massai, M.M.; Minuti, M.; Omodei, N.; et al. Reading a GEM with a VLSI pixel ASIC used as a direct charge collecting anode. Nucl. Instrum. Methods Phys. Res. A 2004, 535, 477-484. [CrossRef]

20. Bellazzini, R.; Angelini, F.; Baldini, L.; Bitti, F.; Brez, A.; Cavalca, F.; Del Prete, M.; Kuss, M.; Latronico, L.; Omodei, N.; et al. Gas pixel detectors for X-ray polarimetry applications. Nucl. Instrum. Methods Phys. Res. A 2006, 560, 425-434. [CrossRef] 
21. Bellazzini, R.; Spandre, G.; Minuti, M.; Baldini, L.; Brez, A.; Cavalca, F.; Latronico, L.; Omodei, N.; Massai, M.M.; Sgro, C.; et al. Direct reading of charge multipliers with a self-triggering CMOS analog chip with $105 \mathrm{k}$ pixels at $50 \mu \mathrm{m}$ pitch. Nucl. Instrum. Methods Phys. Res. A 2006, 566, 552-562. [CrossRef]

22. Bellazzini, R.; Spandre, G.; Minuti, M.; Baldini, L.; Brez, A.; Latronico, L.; Omodei, N.; Razzano, M.; Massai, M.M.; Pesce-Rollins, M.; et al. A sealed Gas Pixel Detector for X-ray astronomy. Nucl. Instrum. Methods Phys. Res. A 2007, 579, 853-858. [CrossRef]

23. Muleri, F.; Soffitta, P.; Bellazzini, R.; Brez, A.; Costa, E.; Fabiani, S.; Frutti, M.; Minuti, M.; Negri, M.B.; Pascale, P.; et al. A very compact polarizer for an X-ray polarimeter calibration. In UV, X-ray, and Gamma-Ray Space Instrumentation for Astronomy XV; SPIE: Bellingham, WA, USA, 2007; Volume 6686, p. 668610.

24. Sakurai, H.; Saito, S.; Masuda, T.; Gunji, S.; Noma, M.; Kanase, G.; Suzuki, Y. The form of X-ray photoelectron tracks in a capillary gas proportional counter. IEEE Trans. Nucl. Sci. 1999, 46, 333-337. [CrossRef]

25. Sakurai, H.; Tokanai, F.; Gunji, S.; Motegi, S.; Toyokawa, H.; Suzuki, M.; Hirota, K.; Kishimoto, S. Measurement of X-ray polarization using optical imaging detector with capillary plate. Nucl. Instrum. Methods Phys. Res. A 2004, 525, 6-11. [CrossRef]

26. Black, J.K.; Baker, R.G.; Deines-Jones, P.; Hill, J.E.; Jahoda, K. X-ray polarimetry with a micropattern TPC. Nucl. Instrum. Methods Phys. Res. A 2007, 581,755-760. [CrossRef]

27. Weisskopf, M.C.; Ramsey, B.; O’Dell, S.; Tennant, A.; Elsner, R.; Soffitta, P.; Bellazzini, R.; Costa, E.; Kolodziejczak, J.; Kaspi, V.; et al. The Imaging X-ray Polarimetry Explorer (IXPE). In Space Telescopes and Instrumentation 2016: Ultraviolet to Gamma Ray; SPIE: Bellingham, WA, USA, 2016; Volume 9905, p. 990517.

28. Costa, E.; Bellazzini, R.; Bregeon, J.; Brez, A.; Frutti, M.; Di Cosimo, S.; Latronicio, L.; Lazzarotto, F.; Matt, G.; Minuti, M.; et al. XPOL: A photoelectric polarimeter onboard XEUS. In Space Telescopes and Instrumentation 2008: Ultraviolet to Gamma Ray; SPIE: Bellingham, WA, USA, 2008; Volume 7011, p. 70110F.

29. Fabiani, S.; Bellazzini, R.; Berrilli, F.; Brez, A.; Costa, E.; Minuti, M.; Muleri, F.; Pinchera, M.; Rubini, A.; Soffitta, P.; et al. Performance of an Ar-DME imaging photoelectric polarimeter. In Space Telescopes and Instrumentation 2012: Ultraviolet to Gamma Ray; SPIE: Bellingham, WA, USA, 2012; Volume 8443, p. 84431C.

30. Fabiani, S.; Costa, E.; Bellazzini, R.; Brez, A.; di Cosimo, S.; Lazzarotto, F.; Muleri, F.; Rubini, A.; Soffitta, P.; Spandre, G. The gas pixel detector as a solar X-ray polarimeter and imager. Adv. Space Res. 2012, 49, 143-149. [CrossRef]

31. Fabiani, S.; Campana, R.; Costa, E.; Del Monte, E.; Muleri, F.; Rubini, A.; Soffitta, P. Characterization of scatterers for an active focal plane Compton polarimeter. Astropart. Phys. 2013, 44, 91-101. [CrossRef]

(C) 2018 by the author. Licensee MDPI, Basel, Switzerland. This article is an open access article distributed under the terms and conditions of the Creative Commons Attribution (CC BY) license (http:/ / creativecommons.org/licenses/by/4.0/). 